\title{
MICROSTRIP ANTENNA CIRCUIT MODEL AND LINEAR PATTERN CORRECTION
}

\author{
Ilkka Salonen, Clemens Icheln, and Pertti Vainikainen \\ Radio Laboratory \\ IDC, SMARAD, Helsinki University of Technology (TKK) \\ Espoo, Finland \\ ilkka.salonen@tkk.fi
}

\begin{abstract}
Mutual coupling, among other effects, causes the element patterns in an antenna array to be different. In the practical adaptive use of the array identical element patterns are usually expected. Linear pattern correction with matrix multiplication can be used to correct the array element inputs/outputs. In this paper, a multiport model for a microstrip array and a method to find the input circuit for its ports is presented for use in the array correction.
\end{abstract}

\section{INTRODUCTION}

Mutual coupling distorts antenna array element patterns. A matrix method can be used for the correction of the array inputs/outputs $[1,2]$. In addition to mutual coupling also the ground plane edge effect should be included in the correction of a microstrip array [3,4]. If the antenna elements in an array are purely voltage or current driven, the array pattern correction can be done with the impedance or admittance matrix $[1,4]$. A circuit model for an antenna array is of interest for mutual coupling compensation [3]. When only the measured scattering matrix is known, it is a reverse problem.

\section{THEORY}

Mutual coupling in multiport devices can be presented with impedance $(\mathbf{Z})$, admittance $(\mathbf{Y})$ or scattering matrix $(\mathbf{S})$. In this work impedance and scattering matrices are calculated using measured scattering matrices. Impedance matrix elements for a given port are defined as the ratio of output voltage in each port to input current in the given port when all other ports are open circuited with zero input currents. In the admittance matrix measurement the input is voltage and outputs are currents when other ports are short circuited with zero input voltages [5].

In Fig. 1 we see equivalent circuits for two-element impedance and admittance matrix measurements [6-7]. It is simple to prove, that these circuits are in accordance with impedance and admittance matrix definitions and can be both presented a priori as circuit models for a linear passive two-port. These models can give for an antenna at a given port an interpretation of isolation, without mutual coupling, when the other ports are open- or short-circuited. [1,6]. The principal difference between these circuits is, that in the case of coupling $Z_{i i}$ and $1 / Y_{i i}$ are different and cannot at the same time present the same "isolated" antenna resonator.

In general, the components in circuits of Fig. 1 can be also complicated circuit structures. One complication to real linear circuit is, that the mutual component can have as well an unphysical negative resistance as a positive one [6]. Because the circuit is passive the mutual components are only explaining the balance in the system and they cannot be found as a part of the physical array.

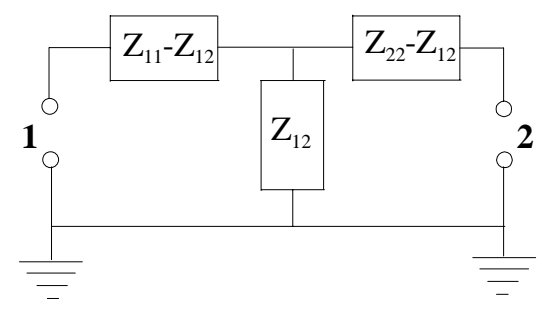

(a)

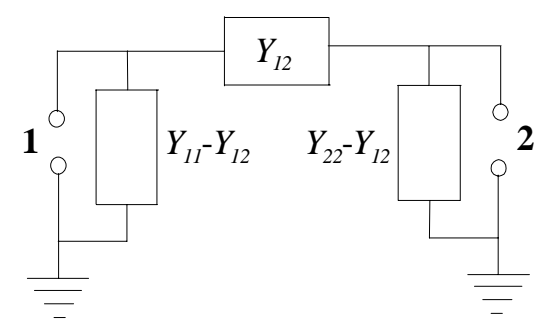

(b)

Figure 1. Two-port circuit models of an antenna pair a) for impedance matrix with a T-circuit [6] and b) for an admittance matrix with a $\pi$-circuit. Impedances $Z_{11}$ and $Z_{12}$ are defined with input current in port 1 with port 2 opened, without a current. Admittances $Y_{11}$ and $Y_{12}$ are defined with input voltage in port 1 and port 2 short circuited with zero voltage.

Usually the signal to an antenna is coming from a voltage generator. In the case without mutual coupling the form of the RF signal vector to/from an antenna array is arbitrary and it can be as well the voltage $(\overrightarrow{\mathbf{V}})$, current $(\overrightarrow{\mathbf{I}})$ or voltage wave vector $\left(\overrightarrow{\mathbf{V}}^{+}\right)$, because without mutual coupling these parameters at a given port depend only on each other and the corresponding matrix dependency between corresponding signal vectors $\left(\overrightarrow{\mathbf{V}}, \overrightarrow{\mathbf{I}}, \overrightarrow{\mathbf{V}}^{+}\right)$is a diagonal matrix. In the case of mutual 
coupling the matrix dependency of a pair of different signal vectors is not diagonal. For an antenna array, the $\mathrm{RF}$ signal at an antenna port is usually the current or voltage (electric field) in the antenna element of the array. When the RF signals at antenna ports are in the form of a voltage wave vector $\mathbf{V}^{+}$the input/output coefficients can be corrected to the voltages or currents by multiplying with corresponding correction matrices $[1,2,4,7,8]$

$$
\begin{aligned}
& \overrightarrow{\mathbf{V}}_{\text {correct }}^{+}=(\mathbf{I}+\mathbf{S})^{-1} \overrightarrow{\mathbf{V}}_{\text {wanted }}=(\mathbf{I}+\mathbf{y}) \overrightarrow{\mathbf{V}}_{\text {wanted }} / 2 \\
& \overrightarrow{\mathbf{V}}_{\text {correct }}^{+}=(\mathbf{I}-\mathbf{S})^{-1} Z_{0} \overrightarrow{\mathbf{I}}_{\text {wanted }}=(\mathbf{I}+\mathbf{z}) Z_{0} \overrightarrow{\mathbf{I}}_{\text {wanted }} / 2,
\end{aligned}
$$

where $\mathbf{y}$ and $\mathbf{z}$ are normalized admittance and impedance matrices, $\mathbf{I}$ is the unity matrix and $Z_{0}$ is the generator impedance used in normalization. In (1) and (2) it is assumed, that the wanted input vectors are the wanted complex weights of the uncoupled element patterns, defined without taking into account the mutual coupling in the antenna array. We see, that the corrections with (1) and (2) have opposite effects on the inputs/outputs due to different signs of the scattering matrix. The mutual coupling compensation removes internal reflections, which cause ripples in the antenna element patterns. If the input/output RF signals are the currents and they should be voltages in the array (or vice versa) then the impedance (admittance) matrix of the array is the corresponding correction matrix. The impedance, admittance and scattering matrices are related to each other [5]

$$
\begin{aligned}
& \mathbf{z}=(\mathbf{I}+\mathbf{S}) \cdot(\mathbf{I}-\mathbf{S})^{-1} \\
& \mathbf{S}=(\mathbf{z}+\mathbf{I})^{-1} \cdot(\mathbf{z}-\mathbf{I}) \\
& \mathbf{y}=Z_{0} \mathbf{Y}=Z_{0} \mathbf{Z}^{-1}=\mathbf{z}^{-1} .
\end{aligned}
$$

In modern antenna technology the antenna elements and the input ports devices have dimensions that are not small compared with the wavelength. In this case the feed element should also be presented as an input circuit to be extracted from the array multiport circuit. When higher frequencies with increased bandwidth are used (which is the tendency of radio system evolution), then the problems with input circuits will grow. If there are well known and well modeled feed structures for the antenna elements, then they can be easily extracted. If we have scattering matrix measurements, then we should change to $\mathbf{z}$-matrix presentation when subtracting an impedance component at input and to $\mathbf{y}$-matrix presentation when subtracting an admittance component at input,

$$
\begin{aligned}
& \mathbf{y}_{i}=\mathbf{y}_{i+1}^{i n}+\mathbf{y}_{i+1} \\
& \mathbf{z}_{k}=\mathbf{z}_{k+1}^{i n}+\mathbf{z}_{k+1},
\end{aligned}
$$

,where $\mathbf{y}_{i}$ and $\mathbf{z}_{k}$ are the admittance and impedance matrices at extracting step $i$ and $k(k \neq i)$. Admittance and impedance matrices $\mathbf{y}^{\text {in }}$ and $\mathbf{z}^{\text {in }}$ are diagonal ones and are to be subtracted. Matrices $\mathbf{y}_{i+1}$ and $\mathbf{z}_{k+1}$ are the admittance and impedance matrices after extracting input components. After subtracting an input circuit element the remaining multiport circuit should be closer to an ideal voltage/current driven array.

Stepwise we can subtract whole input circuit changing between $\mathbf{y}$ - and $\mathbf{z}$-presentations, in analog to working with the Smith chart in one-dimensional case, and then use finally the required correction. After that we should go stepwise backward to the original measurement reference points defining a new correction matrix at each step to obtain the final correction matrix for the reference planes of the measured scattering matrix. The reference plane in the input line for the original measurement is usually at the array connectors. If we extract as an example first a parallel input circuit component (diagonals of $\mathbf{Y}_{1}^{i n}=\mathbf{y}_{1}^{i n} / Z_{0}$ ) and next a serial input circuit component (diagonals of $\mathbf{Z}_{2}^{\text {in }}=Z_{0} \mathbf{z}_{2}^{\text {in }}$ ) and use the admittance presentation for the correction in Equation (1), then the correction matrix $\mathbf{K}$ would be

$$
\begin{aligned}
& \mathbf{K}=\left(\mathbf{I}+\mathbf{S}_{m}\right)^{-1} \mathbf{z}_{1} \mathbf{y}_{2}= \\
& =\left(\mathbf{I}+\mathbf{S}_{m}\right)^{-1}\left(\mathbf{y}_{m}-\mathbf{y}_{1}^{i n}\right)^{-1}\left[\left(\mathbf{y}_{m}-\mathbf{y}_{1}^{i n}\right)^{-1}-\mathbf{z}_{2}^{i n}\right]^{-1},
\end{aligned}
$$

where $\mathbf{S}_{\mathrm{i}}, \mathbf{z}_{\mathrm{i}}$ and $\mathbf{y}_{\mathrm{i}}$ are defined at the same reference point and related to each others by Equations (3)-(5). $\mathbf{S}_{\mathrm{m}}$ is the measured scattering matrix and $\mathbf{y}_{\mathrm{m}}$ is the corresponding normalized admittance matrix for $\mathbf{S}_{\mathrm{m}}$.

\section{RESULTS}

In Fig. 2 we see a multiport circuit model, which is in accordance with admittance matrix definition. The principle is that each port is connected to all other ports and that the admittance at the given port is the selfadmittance minus all mutual admittances for that port. The corresponding circuit model for the impedance matrix can not be determined when the number of ports is more than two. In [9] is presented a $3 \times 3$ multiport, which is not symmetric and does not give all mutual components correctly according to impedance matrix definition, and as following, can be used only as an approximation. We can assume, that the topology/structure in Fig. 2 is the only one explaining mutual coupling exactly in a passive multiport. Thus, the same structure should be used also for the impedance matrix utilizing the dualism of electromagnetics with magnetic circuits [10-13].

Another alternative would be to use mutual components depending parametrically on other circuits as is done traditionally with mutual inductance or capacitance, and also for mutual resistances [14]. The mutual component can be presented for $\mathbf{y}$ - and $\mathbf{z}$-matrices as an internal source of current or voltage depending parametrically on the input to another port. A disadvantage of the passive circuit models without parametric com- 
ponents in Figs. 1 and 2 is, that the mutual component appears there three times.

In general there is no simpler exact circuit model in accordance with the measured admittance matrix than the one shown in Fig. 2. Only when two elements are far from each other the mutual admittance between them can be ignored and the model can be simplified. Each measurement of an impedance matrix component can be modeled with the circuit in Fig. 1a with all other ports open-circuited, but these measurements cannot be combined to a multiport model. This is a serious limitation, because in a linear array each element has usually two neighboring elements.

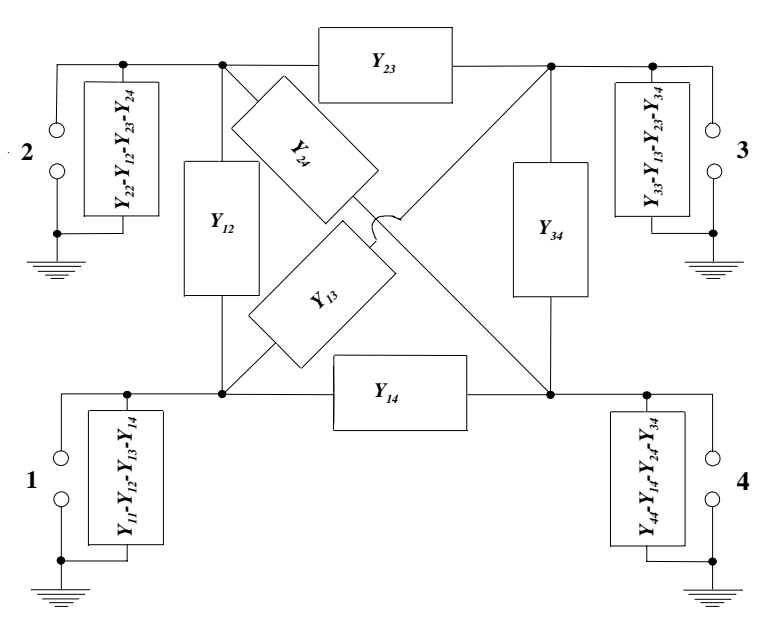

Figure 2. A circuit model defining admittance matrix for a 4-port multiport (for example, for a 4-element array).

A parallel resonator model has been used to explain the behavior of microstrip antenna elements [15-18]. The correction results in [3] using (1) are in accordance with the parallel model. In Fig. 3 we see the measured resonators, when the scattering matrix reference point is at the beginning of the feed probe of a coaxial SMA connector. In the correction done in [3] the reference point is shifted from the level presented in Fig. 3 further $23^{\circ}$ towards the array resulting in a $45^{\circ}$ shift on the Smith diagram. The position of the resonators are after that the same as the position of the ideal admittance resonator in Fig. 3. In the first case (Fig. 3a) the measured $S_{i i}$ :s are presented. The frequency range is 3 ... $6 \mathrm{GHz}$. The frequency of $5.3 \mathrm{GHz}$ is shown for each resonance with a small circle. Reflection coefficients $\rho_{i}\left(y_{i i}\right)$ and $\rho_{\mathrm{i}}\left(z_{i i}\right)$ are the reflection coefficients corresponding to diagonal elements of $\mathbf{y}$ and $\mathbf{z}$ (calculated as in the one-dimensional case). We see, that the resonators of $\mathbf{z}$-presentations are less ideal than the others. For an array with element spacing $0.3 \lambda$ this effect is more pronounced. This shows, that the $\mathbf{y}$-presentation is potentially better for the correction, probably, because it is closer to the ideal circle.

Further, an input circuit to be extracted has been determined. The criterion for a good input circuit is, that after extraction the remaining $\rho_{i}\left(y_{i i}\right)$-resonances, i.e. the reflection coefficients of the admittance matrix diagonal values, are identical and that they are close to the circle of the ideal resonator (see Fig. 3). Different input

a)

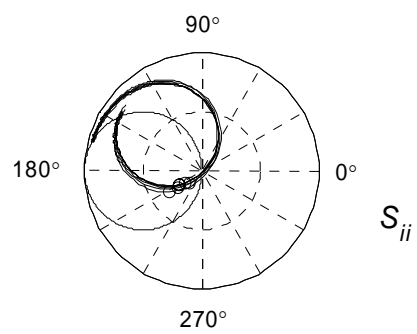

b)
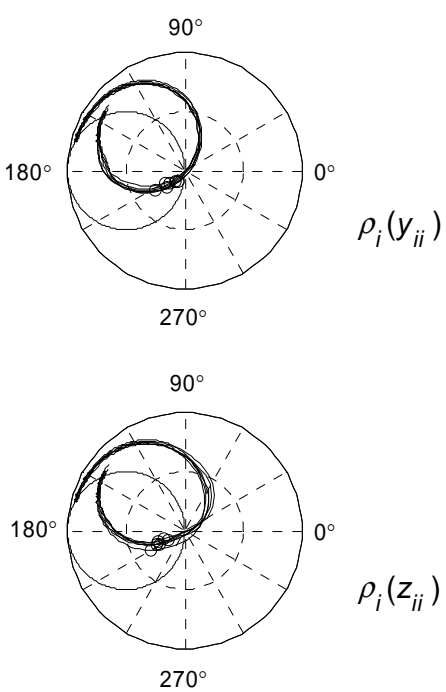

Figure 3. The resonator behavior of an 6-element microstrip array with element spacing of $0.4 \lambda$. Measured $S_{i i}$ :s are shown in a) and in b) and c) are shown the calculated $\rho_{\mathrm{i}}$ :s of $\mathbf{y}$ - and $\mathbf{z}$-presentations, respectively. The perfect circles are for ideal parallel resonators with constant capacitance and inductance and with the resistance of the generator.

circuit structures with up to 6 lumped elements were considered, and three simplest cases with the smallest number of elements were chosen. The general structure is shown in Fig. 4 and the element values for the three cases are given in Table I.

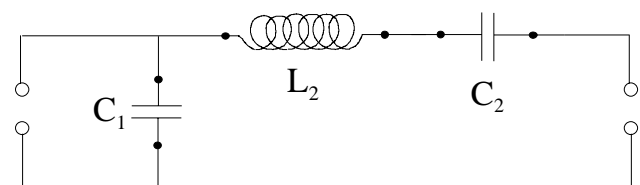

Figure 4. Components of the input circuit.

The resulting pattern corrections in these cases are about the same as those in [3] and [4], where the reference plane was redefined and only a scattering matrix or its eigenstructure was used. In the cases A, B and C the resonance circles are moved to the location of the ideal resonance circle. In case $\mathrm{C}$ the resonance circles are smaller than in the other cases, but it is realistic. An input circuit consisting of a series inductance and a parallel capacitance is like a reference plane shift in a 
waveguide done in [3]. The latter is much simpler to do, though. In case $\mathrm{C}$ the correction results for some arrays are better than for cases A and B. But the improved pattern correction is not necessarily a good criterion for a better input circuit. Antenna patterns in a microstrip array are perturbed also by the ground plate edges [3], and thus false input circuits might also give good correction results.

Table I Input circuit component values

\begin{tabular}{|c|c|c|c|}
\hline & $\mathrm{C}_{1}$ & $\mathrm{~L}_{2}$ & $\mathrm{C}_{2}$ \\
\hline $\mathrm{A}$ & - & $0.60 \mathrm{nH}$ & - \\
\hline $\mathrm{B}$ & - & $0.79 \mathrm{nH}$ & $5 \mathrm{pF}$ \\
\hline $\mathrm{C}$ & $0.5 \mathrm{pF}$ & $0.50 \mathrm{nH}$ & - \\
\hline
\end{tabular}

Another criterion used to find a suitable input circuit is the behavior of the mutual matrix components. Mutual admittances $y_{i j}$ :s for equidistant array element pairs should be nearly identical. Also, $y_{i j}$ :s should oscillate regularly [6]. The case B removes a peak in $y_{i j}$ at 3.8 $\mathrm{GHz}$ with a minor effect on the pattern correction at 5.3 GHz. However, it allows to find $\mathrm{L}_{2}$ and $\mathrm{C}_{2}$ more accurately. In case $\mathrm{C}$ different pairs of $\mathrm{C}_{1}$ and $\mathrm{L}_{2}$ give the same result when matching the resonant circles. This results in unwanted uncertainty. From this example we see, that the complexity of the pattern correction using the admittance matrix with input circuit extraction exceeds that of earlier corrections using scattering matrix $[3,4]$.

\section{CONCLUSIONS}

It was demonstrated, that the matrix correction of an antenna array can be done conveniently, in the case that the array circuit model is one of the idealized models with voltage or current driven antenna elements. A possible input circuit can be extracted. Further, in general case the corresponding linear electric circuit model cannot be found for the impedance matrix and instead a magnetic circuit model would be needed to explain the mutual coupling in an array. For a microstrip array the admittance matrix presentation is preferable and for it the exact equivalent electric circuit can be found also in the case of several elements. The extraction of the input circuit can give better pattern correction results than the reference plane shift in a waveguide, but is a more complicated method.

\section{REFERENCES}

[1] I. Gupta and A. Ksienski., "Effect of mutual coupling on the performance of adaptive arrays", IEEE Trans. Ant. Prop., vol. AP-31, Sep. 1983, pp. 785-791.

[2] H. Steyskal and J. Herd, "Mutual coupling compensation in small array antennas", IEEE Trans. Ant. Prop., vol. 38, Dec. 1990, pp. 1971-75.
[3] I. Salonen, A. Toropainen, and P.Vainikainen, "Linear pattern correction in a small microstrip antenna array," IEEE Trans. Ant. Prop., vol. 52, No. 2, Feb. 2004, pp. 578-586.

[4] I. Salonen, "Pattern distortion and impedance mismatch in small microstrip antenna arrays", Licentiate Thesis, Helsinki University of Technology, Department of Electrical and Communications Engineering, 2002, 170 p.

[5] D. M. Pozar, Microwave engineering, New York, John Wiley \& Sons inc., 1998, 716 p.

[6] J. D. Kraus, Antennas, McGraw-Hill book company, second edition, New York 1988, 892 p.

[7] W. K. Kahn, "Impedance-match and element-pattern constraints for finite arrays", IEEE Trans. Ant. Prop., vol. AP-25, Nov. 1977, pp. 747-755.

[8] L. Pettersson, M. Danestig, and U. Sjöström, "An experimental S-band digital beamforming antenna", IEEE AES Systems Magazine, Nov. 1997, pp. 19-27.

[9] R. Levy, "Derivation of equivalent circuits of mictowave structures using numerical techniques", IEEE Transactions on microwave theory and techniques, vol. 47, No. 9, Sep 1999, pp. 1688-1695.

[10] N. C. Cheung, and K. K-C. Chan, "Magnetic modeling of a mutually coupled variable reluctance gripper", $28^{\text {th }}$ annual conference of the Industrial electronics society (IECON 2002), vol. 4, Nov. 2002, pp. 2733-2738.

[11] M. A. Preston, J. P. Lyons, "A switched reluctance motor model with mutual coupling and multi-phase excitation", IEEE Transactions on magnetics, vol. 27, Nov. 1991, pp. 5423-5425.

[12] L. J. Giacoletto, "Magnetic circuits analysis using electronic circuit analysis program", Proceedings of conference on Power electronics in transportation, Dearnborn, MI USA, 1994, pp. 91-94.

[13] A. H. Mohammadian, N. M. Martin, and D. W. Griffin, "A theoretical and experimental study of mutual coupling in microstrip antenna arrays", IEEE Trans. Ant. Prop., vol. 37, Oct. 1989, pp. 1217-1223.

[14] H. Ymeri, B. Nauwelaers, K. Maex, S. Vanderberghe, and David de Roest, "New analytical expressions for mutual inductance and resistance of coupled interconnects on lossy silicon substrate", Digest of Papers of 2001 Topical Meeting on Silicon Monolithic Integrated Circuits in RF Systems, Ann Arbor, MI USA, 2001, pp. 192-200.

[15] K. R. Carver and J. W. Mink, "Microstrip antenna technology", IEEE Trans. Ant. Prop., vol. AP-29, Jan. 1981, pp. 3-25.

[16] W. F. Richards and Y. T. Lo, "An improved theory for microstrip antennas and applications", IEEE Trans. Ant. Prop., vol. AP-29, Jan. 1981, pp. 38-46.

[17] B. Robert, T. Razban, and A. Papiernik, "Capasitors provide input matching of microstrip antennas", Microwaves $\& R F$, vol. 33, July 1994, pp. 103-106.

[18] V. Voipio, J. Ollikainen, and P. Vainikainen, "Quarterwave patch antenna with $35 \%$ bandwidth", IEEE Ant. Prop. Soc. Int. Symp., 1988, pp. 790-793. 